\title{
TREATMENT OF TUBERCULOSIS OF THE KNEE
}

\author{
F. HaRwood Stevenson, M.D., M.R.C.P.
}

Consultant Physician, Royal National Orthopadic Hospital, and the Institute of Orthopadics, London.

A FEW general observations are pertinent before considering the treatment of tuberculosis of the knee in its different clinical manifestations.

1. Tuberculosis of a joint implies that blood-stream dissemination following primary infection has occurred. Full investigation of the patient with regard to the lungs, the urine and other bones and joints is therefore essential.

2. Monarticular rheumatoid arthritis in adults or children is by no means uncommon and may exist in a child who already has a positive Mantoux, so that the diagnosis of an early case without bone destruction may require considerable investigation.

3. The range of clinical material and the incidence vary much in different parts of the world. In England today tuberculosis of the knee is rarely seen, is frequently seen early and has become relatively much less common in children. In many countries where there are still numbers of sputum-positive patients and a lower standard of living and medical care, and where communications are difficult, many patients and more with advanced disease are seen.

4. The results of some of our diagnostic tests vary with the race of the patient. This is particularly true of the sedimentation rate. Natives of Britain with active skeletal tuberculosis commonly have a sedimentation rate which is, if raised at all, between 15 and $35 \mathrm{~mm}$. in the first hour. Much higher figures than this in a spinal lesion raise a strong suspicion that the disease is staphylococcal and not tuberculous; similar high figures in a patient with a single joint affected would suggest rheumatoid disease. However when we meet patients in this country from Asia, Africa or the West Indies with a closed single tuberculous lesion, they commonly have a sedimentation rate in the higher range when first seen, e.g., $50-70 \mathrm{~mm}$. in the first hour.

5. Radiographically it is most important to take strictly comparable views of the sound knee, if possible on the same film, so as to aid the detection of minor degrees of osteoporosis and changes in texture of the cancellous bone.

Before the era of antibiotics it was taught that spontaneous or deliberate arthrodesis was the only safe end-result in tuberculosis of the knee. Usually despite prolonged rest varying degrees of bone destruction occurred, with fibrous adhesions and restriction of movement. Such joints were always at risk of re-activation by minor degrees of trauma. The natural resistance of the human body now aided by antibiotics may ultimately produce quiescence of the lesion. We have then created a desert for the mycobacteria inhabiting the lesion, but he would be bold who asserted that nowhere in the areas of "healed" and probably fibrotic tissue werळ there no lurking bacteria or their cocca forms. Blood and effusion provide the ideaP watering of the desert, and it is for this reason that the tearing of an adhesion in joint with limited movement can so easily re-activate tuberculosis.

But with the new drugs, with or without operation, we can commonly hold the situation if a diagnosis is made early enough, and this gloomy outlook is no longer with us.

\section{Clinical Types}

Clinically cases of tuberculosis of the knee can be divided into a number of groups depending upon the stage the disease has reached before diagnosis:

1. Effusion into the joint, with perhaps some thickening of the synovial membrane, with radiologically a good joint space and no evidence of a bone focus or any bone erosion or destruction; these joints commonly have a very good or even normal range of flexion, restricted if at all by the size of the effusion.

2. Little or no effusion, in a joint with a long history, with varying degrees of thickening and fibrosis of the synovial membrane, possibly with adhesions preventing full flexion, but still with a reasonably wide joint space and no radiological evidence of bone disease. 
3. Those with synovial disease and a bone focus within the tibia, femur or patella. Such a focus presumably must have at least microscopic communication with the joint space but this type still has a reasonable joint space and radiologically intact joint surfaces.

4. Finally, with a diminished joint space, considerable destruction of the bone surfaces and very considerable loss of range of movement.

Clearly there are various gradations between one group and the next.

\section{Investigations}

Proof of tuberculosis of the knee may be obtained by inguinal gland biopsy, but there is so little damage from a limited anterior synovial biopsy that this is preferable, and besides it is more certain. It is important however not to rely upon any one method of investigation of the biopsy material. The histology may be non-specific and doubtful and must be aided by Lowenstein culture and inserting some of the material into a muscle pouch in the leg of a guinea pig. Examination of unstained sections of the histological material with ultra-violet light may show fluorescing mycobacteria. Naturally a patient will not be considered possibly tuberculous unless known to have a positive Mantoux or Heaf test; with an active lesion it is likely that they will be positive 1 in 5000 or 2 to $4+$, according to the test used, rather than only weakly positive.

\section{Principles of Chemotherapy}

Experience has shown that six to twelve months chemotherapy is adequate in most orthopædic lesions, even though two years chemotherapy is known to be the minimum necessity in chest and renal lesions. The obtaining of material for culture and sensitivity checks against the three main drugs, streptomycin, PAS and INAH is obviously of great importance, particularly when there is the possibility or likelihood of a patient having received the primary infection from a person with resistant organisms. In the first instance triple drug chemotherapy is advisable, because the chance of a mutant developing which is resistant to all three drugs is very slight.

Various antibiotic combinations have been used. Our own preference has been for streptomycin $1 \mathrm{~g}$. once a day for three months, and thereafter $1 \mathrm{~g}$. three times a week; smaller doses in proportion of course are given for children, for whom we have used $0.75 \mathrm{~g}$. between the ages of 5 and 10 years, below 5 years $0.5 \mathrm{~g}$. and below 2 years $0.25 \mathrm{~g}$. PASo $5 \mathrm{~g}$. three times a day for an adult seems. adequate from the many trials which have been done, and in any case this is as much as? most patients can tolerate. There is some evidence from the M.R.C. trials in Madraso (1960) that INAH is best given in one single? dose daily in order to give a peak blood level? and that $300 \mathrm{mg}$. is an adequate dose evenes for patients who metabolise INAH rapidly. $\overrightarrow{0}$ The dosages of PAS and INAH are not changed throughout the course. Most of our patientsw receive a total of six months to nine months of treatment. If for any reason such as otos toxicity or other difficulty the streptomycin has. to be stopped, PAS and INAH are continued? At that dose of INAH it is wise to giveo pyridoxine (vitamin $B_{6}$ ) $20 \mathrm{mg}$. once daily to guard against the excess pyridoxine excretiont during INAH therapy which can result in ao polyneuritis.

Antibiotics for use when any of the standardes three cannot for some reason be used or ifo the organism is known to be resistant to $a_{\overrightarrow{0}}^{\mathrm{D}}$ drug are:-

(a) Viomycin: Adult dose 1 g. b.d. i..${ }^{\infty}$ on two days a week, i.e., 4 g. a week. It ${ }_{1} s_{\circ}$ potentially toxic to the 8th nerve so should nōit $t^{+}$ replace streptomycin if that drug has been stopped because of ototoxicity.

(b) Pyrazinamide: Is a potentially hepato-o toxic drug but forms a very good combination with INAH. Adult dosage 0.5 g. t.d.s but å serum G.P. transaminase level should be $\overrightarrow{\vec{O}}$ checked weekly or at least fortnightly. Any rise 3 to over $40 \mathrm{Karmen}$ units $/ \mathrm{ml}$. gives warning of impending liver damage before the development ${ }^{\circ}$ of jaundice and the drug of course should be훅 immediately and permanently stopped.

(c) Ethionamide in oral dosage 0.25 g. b.d. to q.d.s. varying with body weight. Some gastrointestinal intolerance can occur.

All these three drugs, like all anti-tuberculous drugs, should of course not be used alone.

Intra-articular Chemotherapy. Whether there is an effusion it is easy to use intra-articular chemotherapy. The presence of an effusion is stressed, because if there is only a thickened ${ }^{N}$ œedematous or fibrotic synovium the insertion $N_{\omega}$ of a needle and the injection of fluid willo produce bleeding and trauma and possible re-o activation. A needle into the joint space does $\bar{\varnothing}$ no harm. The effusion can be removed and? then another syringe with $1 \mathrm{~g}$. of streptomycin ${ }_{0}$ attached to the same needle for the injection. $\frac{+}{0}$ 
When this is done twice a week-instead of the intramuscular injection on these days - the effusion tends to persist, but in our experience at the end of three months when one ceases the intra-articular injections and simply continues the intramuscular injections three times a week the effusion rapidly clears.

In patients with minimal and purely synovial involvement with effusion, good joint space, and good movement, the disease may have been present for a number of years, with a history of repeated effusions following minor trauma but clearly the degree of infection is still minimal. These joints stiffen rapidly if splinted in plaster casts. They require chemotherapy and do well with intra-articular streptomycin. The presence of the effusion facilitates this. These patients should always be allowed free movement in bed unless too young to obey orders not to stand up, when a Thomas's splint for two or three months may have to be used.

Some case histories with comments will illustrate the principles of treatment in the several groups:

\section{Type 1. Synovial involvement with effusion:}

W.L., aged 43, male. T.B. glands treated 1953 with 50 injections of streptomycin and six months PAS and INAH. June 1963: one year history of ache in right knee, six months swelling, three months increasing pain and stiffness. Acid fast bacilli in the synovial fluid and culture positive: sensitive PAS and INAH but slightly resistant to streptomycin at $4 \mathrm{mg} / \mathrm{ml}$. A plaster cast was applied in another hospital when flexion was possible to 90 degree. When the plaster was removed after one month, he could hardly flex the knee at all. There was an effusion but radiologically the knee was normal apart from slight osteoporosis.

He then lay free in bed without plaster. $1 \mathrm{~g}$. streptomycin intra-articularly twice weekly and on other days $1 \mathrm{~g}$. intramuscularly. Oral PAS 5 t.d.s. and INAH $300^{\circ} \mathrm{mg}$. Once daily.

After two weeks flexion to 40 degrees, and at two months, 70 degrees. After three months, sterptomycin PAS and INAH continued but with streptomycin intramuscularly only and reduced to three times a week. At the end of four months, streptomycin stopped because of slight deafness and PAS and INAH continued for a further six months.

At five months flexion to 90 degrees, and at seven months flexion 100 degrees with full extension. The effusion cleared after the end of the intra-articular course. At fourteen months: no effusion, no symptoms, flexion 120 degrees, extension full with full activity.

Stevenson, Cholmeley and Jory (1958) reported that of 24 patients with purely synovial disease 14 ended with full mobility, seven with 95 to 135 degree of flexion and another two with 70 to 90 degree. The majority of these did not receive intra-articular chemotherapy, but with an effusion intra-articular streptomycin is simple, safe and helpful. In the above case particularly, one felt that the higher local concentration of streptomycin was valuable in combating an organism moderately resistant to it.

We have seen several patients in this group recently who have lost much of their flexion movement as a result of unnecessary fixation by plaster of Paris; not all recovered as well as this man.

Type 2. Thickened fibrotic synovium with little or no effusion but a reasonable joint space:

These joints commonly have a very limited range of flexion and are consequently in danger from minor trauma when walking on irregular surfaces or on stairs, etc. If the range of movement does not improve in the first two months of chemotherapy with bed rest without splinting, then even if there is no evidence of involvement of the bone, an arthrotomy with anterior synovectomy may give the only chance of increasing joint function.

Case History. Synovial disease, thickened synovium without effusion:

B.M., aged 14, male. In September, 1962 he was kicked on the knee, which became swollen and tender. In April, 1963 the knee was still swollen and beginning to stiffen in the morning and he was limping. In June, 1963 the knee was aspirated and a biopsy in September was histologically positive. He was put into a plaster cylinder and on admission to hospital in October, 1963 had only 20 degrees of movement, no effusion and a thick boggy synovial membrane but a mobile patella. Intra-articular streptomycin was not attempted but he was given daily 1 g. i.m. with oral INAH $300 \mathrm{mg}$. once daily and PAS 5 g. t.d.s. He was allowed freedom in bed. The range of flexion rapidly improved to 80 degrees and by December, 1963 to 135 degrees. In the second three months triple drug chemotherapy was continued with the streptomycin reduced to three times weekly.

\section{Open operation:}

At this point it is pertinent to discuss the place of open operation, synovectomy, curettage of juxta-articular bone lesions and in general what has been also called débridement of the diseased joint. One of the best papers on this subject is that of $M$. C. Wilkinson (1962). He described 73 cases of tuberculosis of the knee treated by chemotherapy and in 39 cases synovectomy with débridement was judged necessary and performed. Wilkinson states that with loss of joint space and marginal erosions or bone destruction within the joint open operation is necessary. He first gives six to ten weeks treatment without intra-articular streptomycin and before and after operation uses a Thomas's splint until the joint is cool and the swelling almost gone. His operative procedure is to remove the diseased synovium from the anterior and posterior parts of the joint and from the supra-patellar pouch; he 


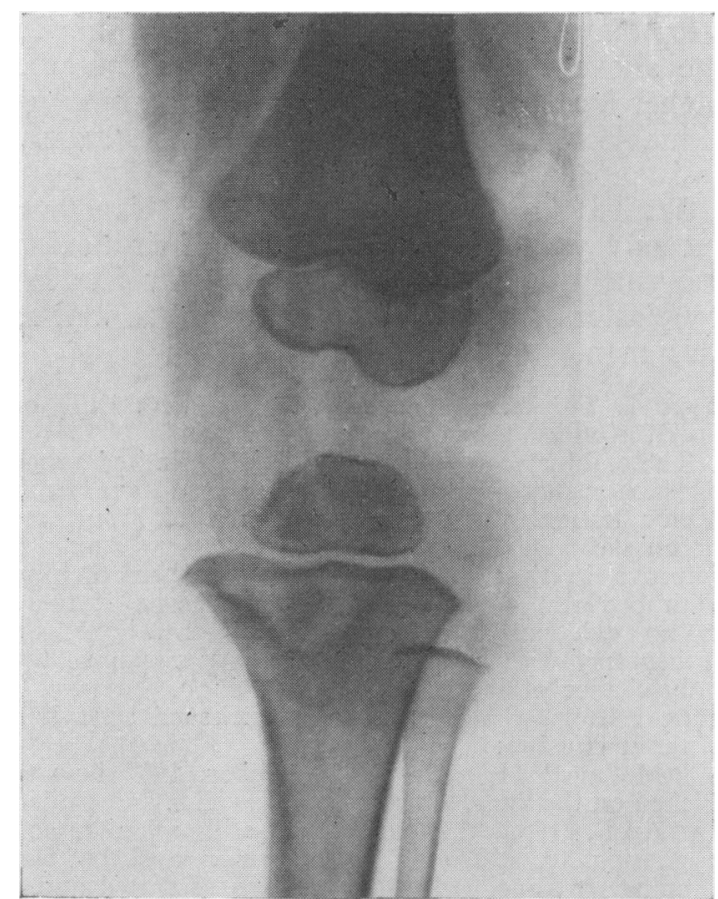

FIG. 1.-G.M. Extra-articular lesion with sequestrum. Synovial disease also.

removes pannus and also the semilunar cartilages if they are fragmented. He also removes the infra-patellar fat pads because by subsequent fibrosis they contribute to loss of mobility of the joint. He makes a point of removing the tourniquet before closing the joint so as to observe bleeding points and secure perfect hæmostasis. He gives antibiotics for six months. 12 out of 14 patients of this type so treated ended up with 90 degrees or more of flexion, but in 25 adults his results were not so good as regards the amount of flexion, though they were otherwise successful. He points out that with at least 35 degrees of flexion walking is usually possible without too great a risk of catching the toe and tearing adhesions.

Nagano and others (1962) have also advocated joint clearance and six months chemotherapy with three drugs. Out of 39 cases they obtained "good mobility" in 82 per cent. They start exercising the joint two weeks after operation but do not allow weight bearing for at least two months. They state that in children with radiologically normal joints there were good results in such cases without operation. It will be agreed by many now that in the type of case in which there is radiological evidence of intra-articular bone or cartilage disease operative clearance is probably wise,

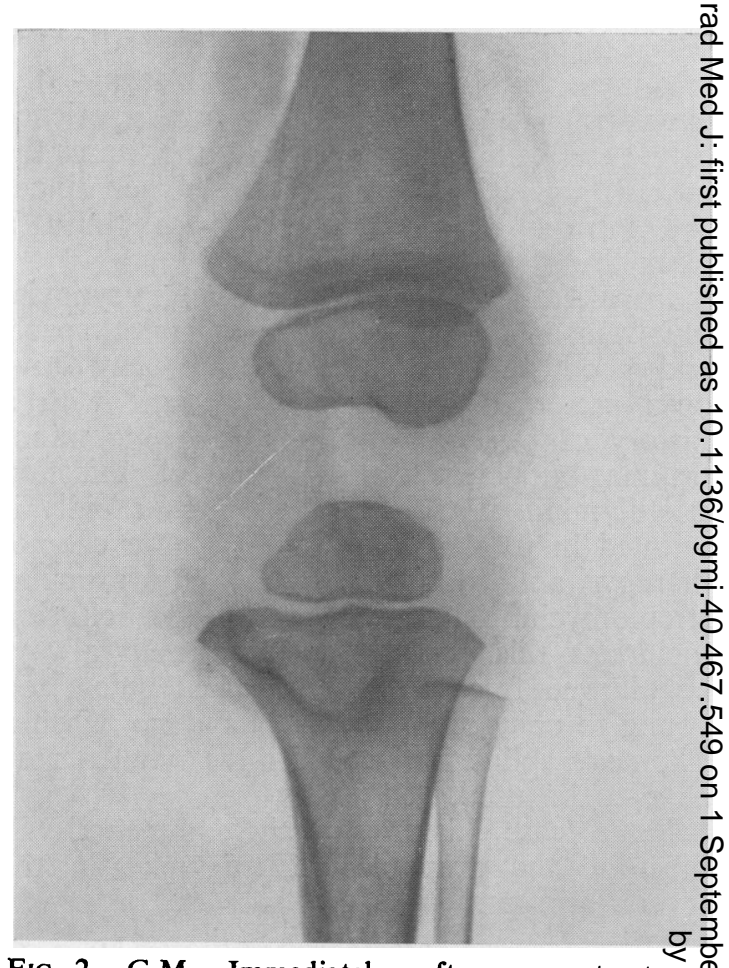

FIG. 2.-G.M. Immediately after sequestrectomy Joint not opened and epiphyseal plate fot touched.

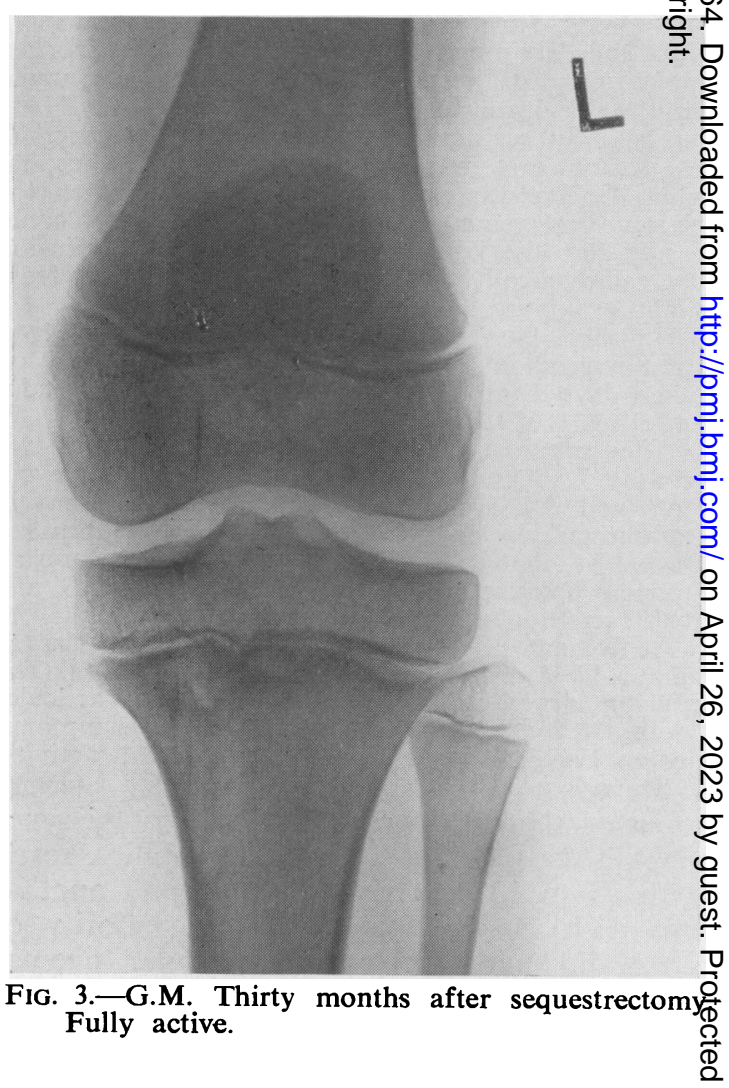



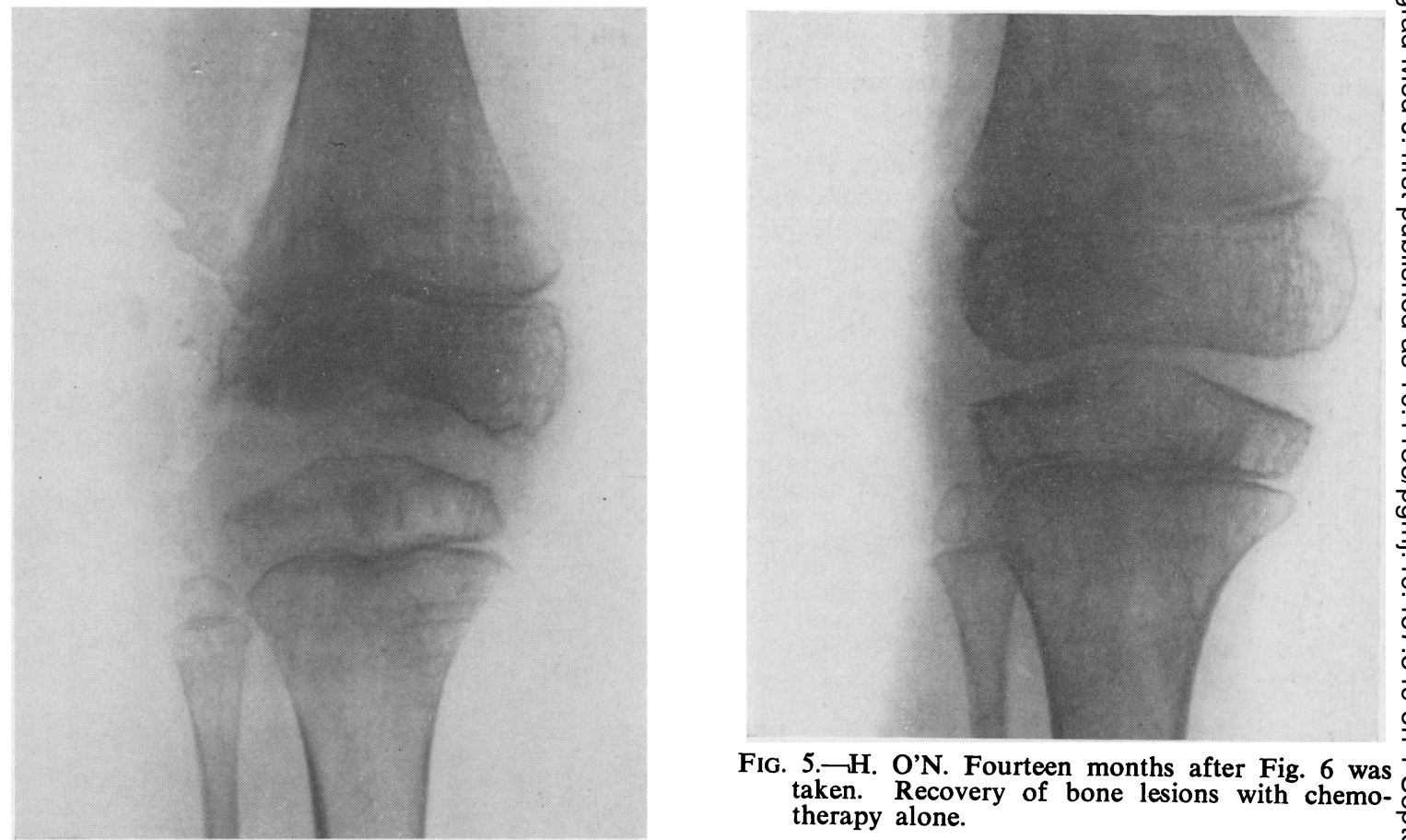

FIG. 5.-H. O'N. Fourteen months after Fig. 6 was taken. Recovery of bone lesions with chemotherapy alone.

Fig. 4.-H. O'N. Synovial tuberculosis and destruction of part of lateral femoral condyle.

even though as in one of the cases to be quoted one may obtain a good result at times without operation.

Type 3 (1): Synovial tuberculosis with extra-articular and juxta-articular bone lesion:

G.M. born 1951, male. At 18 months left knee swollen and child was limping. Large gland in left groin. Radiologically a cavity in metaphysis of tibia with a sequestrum and disease in the epiphysis. (Fig. 1). Inguinal gland biopsy histologically positive November, 1952 commenced $0.25 \mathrm{~g}$. streptomycin b.d. and PAS 0.75 g. t.d.s. January, 1953, sequestrum removed from tibial metaphysis, cavity curetted, and streptomycin powder $1 \mathrm{~g}$. into the cavity. (Fig. 2). Epiphyseal plate seen at top of cavity but not pierced. Pus was guinea-pig and Lowenstein culture positive; organism fully streptomycin sensitive. Following curettage of the cavity INAH (then a new drug) $20 \mathrm{mg}$. t.d.s., i.e., $4 \mathrm{mg}$./kilo. body weight per day was added to treatment and streptomycin changed to $0.5 \mathrm{~g}$. once daily. February, 1953, PAS stopped but other two drugs continued until May, 1953. October, 1953 discharged home with full range of movement. 1958: Best runner in his class at school, with full painless movement.

Fig. 3 shows the knee in 1962, nine years after operation.

Type 3 (2): Intra-articular epiphyseal destructive disease and synovial involvement.

H.O'N, female, born 1945. Trauma at the age of three was followed by symptoms of tuberculosis of the knee in December, 1948. Pus from abscess pointing behind the knee was guinea-pig positive. Streptomycin $0.5 \mathrm{~g}$. daily alone was given for five months, and conservative treatment continued for a

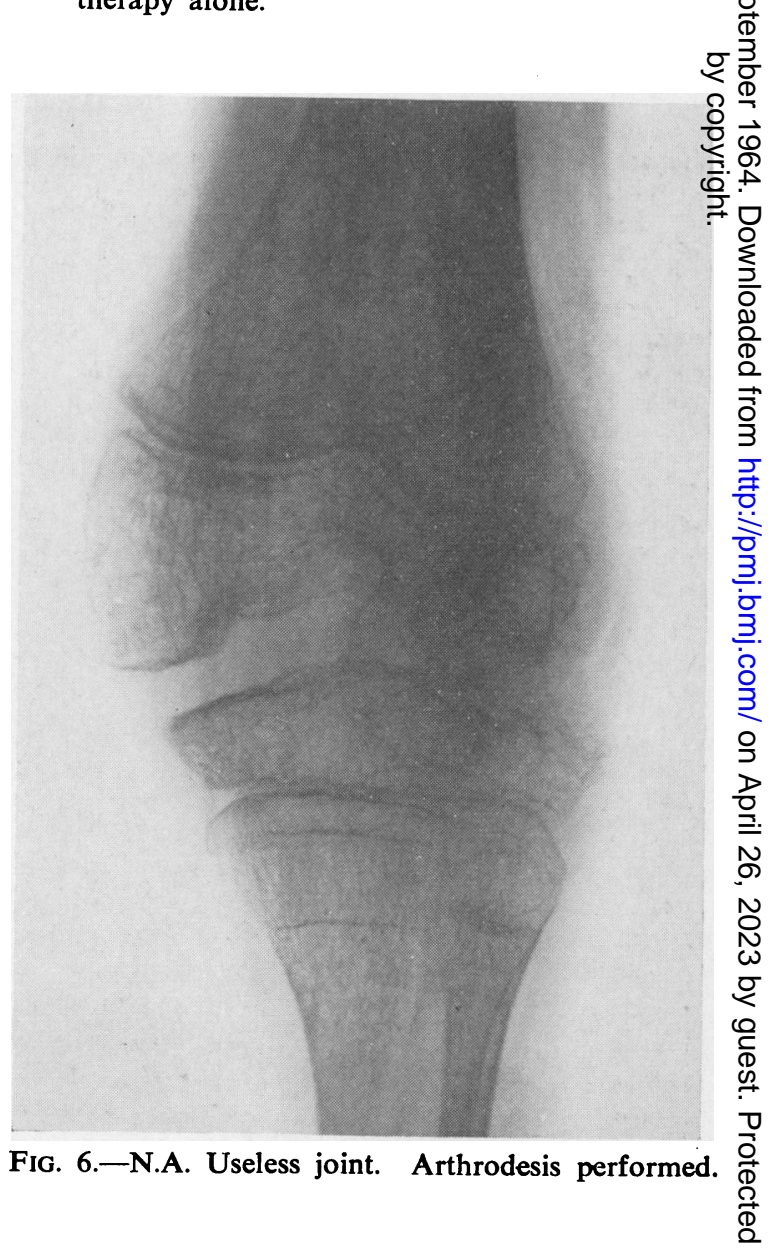


year. At the beginning of the course there was considerable destruction of the lateral condyle of the femur (Fig. 4), but with chemotherapy healing and reformation of the diseased condyle occurred without operation (Fig. 5).

It remains true that really gross joint destruction is best treated by arthrodesis under antibiotic cover. The production of a mobile joint in these circumstances will not occur, or at best adhesions will only allow very slight movement and their tearing will be liable to encourage reactivation.

Type 4: Gross synovial and bone disease.

N.A. born 1948, male. At the age of seven had already a three-year history of tuberculosis of the left knee. He received chemotherapy of doubtful amount in 1954 and in November, 1955 a synovectomy was done. In June, 1956 he was seen (Fig. 6) with a fixed flexion deformity, loss of joint space, and deformity of the femoral condyle. It was agreed that a safe useful mobile joint was not obtainable. A Charnley arthrodesis was performed.
In patients of this type and in those with even grosser destruction of the bone surface, $\bar{z}$ arthrodesis after removal of as much diseased $\underset{\complement}{\AA}$ tissue as possible is the proper procedure. $C$ Chemotherapy should be given with three $\overrightarrow{\vec{s}}$ drugs for at least six months.

\section{Summary}

1. The principles of chemotherapy in ortho- $\frac{\overline{\bar{F}}}{\vec{D}}$ pædic tuberculosis and the management of $\stackrel{\curvearrowright}{\propto}$ of the tuberculous knee in particular are $\frac{\infty}{\infty}$ described.

2. Four broad types of tuberculosis of the knee and the therapeutic approach to each $\overrightarrow{\vec{\omega}}$ are described.

3. The place of synovectomy with débride- $\stackrel{0}{7}$ ment of the joint under chemotherapy cover is described for those patients with $\overrightarrow{0}$ disease more advanced than that of the $\vec{\sigma}$ synovium only but for whom a mobile. joint is still a reasonable hope.

\section{REFERENCES}

Nagano, M., Udagawa, E., Hara, T., Honda, S., OHta, M. (1962): Synovectomy as a Treatment of Tuberculosis $\vec{\mathcal{}}$ of the Knee: Follow-up Studies and a Review of Literature, J. Jap. Orthop. Assn., 35, 1245.

Stevenson, F. H., ChOlmeley, J. A. and JORY, H. I. (1958): Tuberculosis of the Knee: Results and Chemo- $\frac{\vec{D}}{\mathbb{D}}$ therapy between 1948 and 1956, Tubercle, 39, 1 .

TUBerCulosis ChemotheraPy UNIT, Madras (1960): A Concurrent Comparison of Isoniazid plus PAS, with 3 Regimens of Isoniazid alone, in the Domiciary Treatment of Pulmonary Tuberculosis in S. India, Bu丹 Wld. Hlth. Org., 23, 535 . WILKINSON, M. C. (1962): Partial Synovectomy in the Treatment of Tuberculosis of the Knee, J. Bone Jt. Sur
44B, 34. 